\title{
Efecto apalancamiento en el mercado accionario colombiano
}

\author{
Leverage effect on the colombian stock market
}

DOI: http://dx.doi.org/10.21676/23897848.1671

Lizet Viviana Romero-Orjuela

Economista Universidad Nacional de Colombia, Estudiante de la Maestría en Estadística de la Universidad Nacional, Bogotá. Colombia. lvromeroo@unal.edu.co

Alexander Trilleras-Martínez Especialista en Estadística, Matemático Universidad Nacional de Colombia. Estudiante de la Maestría en Estadística de la Universidad Nacional, Bogotá. Colombia. atrillerasmar@unal.edu.co

Tipología: Artículo de Investigación Científica y Tecnológica Fecha de Recibido: Diciembre 10 de 2015 Fecha de Aceptación: Febrero 12 de 2016 Para citar este artículo: Romero 0.L., \& Trilleras, M. A. (2016). Efecto Apalancamiento En El Mercado Accionario Colombiano. Clío América. 10 (19), 43 - 54
Resumen: En este artículo se analiza el efecto apalancamiento en el mercado bursátil colombiano, para dicho fin se usan modelos de la familia ARCH que permitan evaluar si tal efecto está presente en el mercado de acciones, específicamente se usan los modelos no lineales EGARCH y TGARCH. Las series elegidas para realizar dicho análisis son el Índice General de la Bolsa de Valores que es el índice más representativo del mercado de acciones del país, junto con el índice COLCAP. Adicionalmente, se tendrán en cuenta seis de las acciones colombianas que son más transadas en la actualidad. Finalmente se encuentra que el mercado accionario de Colombia sí cuenta con efecto apalancamiento; es decir, las malas noticias tienen un impacto mayor en la volatilidad de los rendimientos financieros.

Palabras Clave: Mercado de acciones, Colombia, Efecto apalancamiento, Modelos ARCH, EGARCH, TGARCH.

JEL: C10, C32, C58, G10

Abstract: In this paper the leverage effect on the Colombian stock market is analyzed, for this purpose ARCH family models are used to evaluate the existence of the effect in the stock market. Specifically TGARCH and EGARCH non-linears models are used. The series chosen for this analysis are the Indice General de la Bolsa de Valores which is the most representative of the stock market, the COLCAP index, and six stocks. Finally we find that the Colombian stock market has leverage effect, ie bad news have a greater impact on the volatility of stock returns.

Keywords: Stock Market, Colombia, Leverage effect, ARCH Models, EGARCH, TGARCH. 


\section{Introducción}

Frecuentemente, muchas de las series económicas y financieras presentan varianzas no homocedásticas, por tal motivo, analizar este tipo de datos implica la implementación de modelos que describan de manera eficiente la variabilidad de las series. Engle, en 1982 propone los modelos ARCH (Modelos autorregresivos con heterocedasticidad condicional) que permiten estimar la duración y la volatilidad de series econométricas, lo cual tiene relevancia al analizar si las predicciones de los precios de una acción son confiables o no (Grajales \& Pérez, 2008).

A partir del surgimiento de los modelos ARCH, se ha generado una gran familia de estos. En el presente trabajo, se implementará el modelo EGARCH propuesto por Nelson (1991), que incluye dentro de su formulación el efecto asimétrico que tiene la variación de precios de un activo en su volatilidad y los modelos TGARCH propuestos por Gourieroux y Zakonian en 1994.

Porras y Valdés (2011) realizan un análisis comparativo implementando los modelos EGARCH y TGARCH para analizar los rendimientos diarios de 30 acciones mexicanas y del Índice de Precios y Cotizaciones (IPC) entre el 7 de diciembre de 2005 y el 1 de agosto de 2011. Para efecto del presente trabajo, se modelará el comportamiento del Índice General de la Bolsa de Valores junto con el índice COLCAP que son análogos al IPC mexicano y seis de las acciones colombianas que son más transadas en la actualidad a través de los modelos TGARCH y EGARCH, y así evaluar la existencia del efecto apalancamiento en las acciones colombianas, siendo esta la hipótesis a evaluar.

\section{Metodología}

La volatilidad no constante de las series financieras exige de nuevos modelos que sean capaces de "capturar" efectos de impacto sobre la volatilidad, por ejemplo, los efectos de apalancamiento que generalmente están presentes en las series de rendimientos. Así el documento se organiza como sigue: en una primera etapa se describen de forma general los modelos EGARCH y TGARCH y se justifica su uso en el análisis de series de tiempo financieras; en la segunda etapa, se hace una reseña sobre los trabajos existentes en el modelamiento de series de retornos en Colombia; se continúa con un análisis descriptivo de cada una de las series, haciendo especial énfasis en determinar la presencia de asimetrías y verificando si el ajuste de los datos corresponde a una distribución normal; la cuarta parte corresponde a la implementación de los test pertinentes para establecer si los modelos de varianza condicional son adecuados y de este modo modelar cada una de las series a partir de los modelos EGARCH y TGARCH; al final se realiza una comparación de los resultados de los dos modelos y se da respuesta a la hipótesis establecida sobre la presencia del efecto de apalancamiento en las series que son objeto de análisis, el estudio concluye indicando algunas observaciones generales.

En 1982, Engle propuso un modelo al que denominó ARCH (Autoregressive Conditional Heterosedasticity), un modelo de varianza condicional, donde los supuestos que se tiene sobre la innovación $\varepsilon_{t}$ son modificados permitiendo que $\varepsilon_{t}$ tenga una distribución normal cuya varianza no es constante, esto condicionado a la información $I_{(t-1)}$ (rezagos del error). A partir de esto se establece un proceso estacionario $\sigma_{t}^{2}$, es decir, $\varepsilon_{t} / I_{t-1} \sim N\left(0, \sigma_{t}^{2}\right)$. En general para el modelo $\mathrm{ARCH}, \varepsilon_{t}^{2}$ puede escribirse de la siguiente forma:

$$
\varepsilon_{t}^{2}=\beta_{0}+\beta_{1} \varepsilon^{2}{ }_{t-1}+\beta_{2} \varepsilon^{2}{ }_{t-2}+\ldots+\beta_{q} \varepsilon^{2}{ }_{t-q}+u_{t}
$$

donde $u_{t}$ se distribuye como ruido blanco. Si a la expresión anterior se le toma valor esperado condicional, se obtiene la varianza condicional $\sigma_{t}^{2}$, escrita como sigue:

$$
\sigma_{t}^{2}=\beta_{0}+\beta_{1} \varepsilon_{t-1}^{2}+\beta_{2} \varepsilon_{t-2}^{2}+\ldots+\beta_{q} \varepsilon_{t-q}
$$

Este modelo fue acogido rápidamente y fue la base para la creación de una familia de modelos conocida como modelos GARCH de volatilidad. Cada modelo de la familia GARCH que se propone busca complementar algunas limitaciones de uno anterior, por ejemplo, el modelo GARCH propuesto por Bollerslev (1986), generalizó el modelo ARCH. En dicho modelo se tienen en cuenta no solo las observaciones 
pasadas sino también la volatilidad experimentada por la serie, además resulta ser más parsimonioso que el modelo ARCH.

Para efecto la presente investigación, dado que los modelos ARCH y GARCH no capturan el hecho de que con frecuencia los retornos de un activo están negativamente correlacionados con las variaciones en la volatilidad de los mismos (Reyes \& Sánchez, 2006), esto debido a que la varianza condicional no es capaz de modelar los cambios asimétricos de los incrementos y decrementos de los activos, se implementará el modelo propuesto por Nelson (1991), donde el efecto de asimetría es modelado involucrando una función $g$ donde las innovaciones son independientes e idénticamente distribuidas y con media cero (Casas \& Cepeda, 2008), la relación entre el valor de $Z_{t}$ y su magnitud representada por la expresión $\left|z_{t}\right|-E\left(\left|z_{t}\right|\right)$ se escribe:

$$
g\left(Z_{t}\right)=\theta z_{t}+\lambda\left(\left|Z_{t}\right|-E\left(\left|z_{t}\right|\right)\right)
$$

donde $\theta$ y $\lambda$ son números reales, si se quiere observar el efecto de asimetría, la función $g\left(Z_{t}\right)$ se puede escribir por casos como:

$$
g\left(z_{t}\right)= \begin{cases}(\theta+\lambda) z_{t}-\lambda E\left(\left|z_{t}\right|\right) & \text { si } z_{t} \geq 0 \\ (\theta-\lambda) z_{t}-\lambda E\left(\left|z_{t}\right|\right) & \text { si } z_{t}<0\end{cases}
$$

Porras y Valdés (2011) presentan el modelo EGARCH de la siguiente forma:

$\operatorname{AR}(1)-\operatorname{EGARCH}(1,1)$

$$
\begin{gathered}
r_{i t}=\phi_{i 0}+\phi_{i 1} r_{i t-1}+u_{i t} \\
u_{i t}=\sigma_{i t} \varepsilon_{i t} \\
\ln \sigma_{i t}^{2}=\alpha_{i 0}+\alpha_{i 1}\left(\left|\varepsilon_{i t-1}\right| /\left|\sigma_{i t-1}^{2}\right|\right)+\gamma_{i}\left(\varepsilon_{i t-1} / \sigma_{i t-1}^{2}\right) \\
+\beta_{i} \ln _{i t-1}^{2}(7)
\end{gathered}
$$

Como se observa, al introducir el logaritmo se garantiza que la varianza condicional sea positiva, característica que era necesaria suponer en los modelos anteriores y además el efecto que hace que la volatilidad se comporte diferente frente a innovaciones positivas que frente a negativas (apa- lancamiento) tenga un comportamiento exponencial. El segundo modelo es el modelo TGARCH (threshold GARCH), al igual que el modelo EGARCH es adecuado cuando se tienen series asimétricas. Aquí la desviación estándar condicional depende de los signos de los shocks rezagados; en un sentido práctico, los shocks negativos, es decir las malas noticias, incrementan la volatilidad y ocurre el efecto apalancamiento, lo que sugiere un impacto asimétrico en la serie. Porras y Valdés (2011) proponen el modelo:

$\operatorname{AR}(1)-\operatorname{TGARCH}(1,1)$

$$
\begin{gathered}
r_{i t}=\phi_{i 0}+\phi_{i 1} r_{i t-1}+u_{i t}(8) \\
u_{i t}=\sigma_{i t} \varepsilon_{i t}(9) \\
{\ln \sigma_{i t}^{2}{ }_{i t}^{\wedge}=\alpha_{i 0}+\alpha_{i 1}}\left(\left|\varepsilon_{i t-1}\right|\right) /\left(\left|\sigma_{i t-1}^{2}\right|\right)+\gamma_{i}\left(\varepsilon_{i t-1} / \sigma_{i t-1}^{2}\right) \\
+\beta_{i} \ln \sigma_{i t-1}^{2}(10)
\end{gathered}
$$

donde

$$
I_{i t}= \begin{cases}1 & u_{i t}<0 \\ 0 & u_{i t-1} \geq 0\end{cases}
$$

Se observa que el parámetro esta relacionado con el efecto de apalancamiento, es decir, si se tiene un impacto positivo $\left(u_{i t}>0\right)$, la volatilidad tendrá un efecto $\alpha_{i 1}$ de igual manera si el impacto es negativo (malas noticias) el efecto sobre la volatilidad estará descrito por $\alpha_{i 1}+\gamma_{i^{\prime}}$ si $\gamma_{i}=0$.

Cabe resaltar que la principal diferencia de los modelos EGARCH y TGARCH está en los signos resultantes de los coeficientes $\gamma_{i}$ que determinan el efecto apalancamiento, es decir, para el modelo TGARCH debe suceder que $\gamma_{i}>0$ mientras que en el modelo EGARCH $\gamma_{i}<0$ (Porras \& Valdés, 2011).

Pocos estudios se han realizado con el fin de modelar el comportamiento del mercado accionario colombiano a través de modelos de varianza condicional. Muchos de los mismos se han centrado en el análisis del IGBC, pero no en el comportamiento de las acciones individualmente. Fernández y Pérez. (2006) proponen un modelo $A R(1)-A R C H(2)$ para el rendimiento del Índice de la Bolsa de Valores de Colombia de 2004 a 2006. Charris, García y Parody (2012) trabajan 
igualmente con el IGBC para el período 2008-2012 y encuentran que el modelo más adecuado para predecir el comportamiento de la serie es un $\operatorname{EGARCH}(1,1)$. Se concluye que el efecto apalancamiento está presente en el comportamiento de la serie.

Arjona y Ortiz, 2001 analizan el caso latinoamericano a través del estudio del comportamiento accionario de Argentina, Brasil, Chile, Colombia, México y Venezuela con datos semanales desde 1989 hasta 1994. Para Colombia encuentran pertinente un modelo $\operatorname{EGARCH}(1,1)$, y hallan la existencia de efecto apalancamiento. Porras \& Valdés (2014) usan modelos TGARCH para el mercado accionario latinoamericano y resaltan la conveniencia de usar modelos de varianza condicional que tengan en cuenta los efectos apalancamiento.

Haque, Hassan y Varela (2001) muestran un enfoque similar al establecido por los autores anteriores a través de un modelo GARCH $(1,1)$ para las series del índice del mercado de acciones del IFC de Brasil, Chile, Colombia, México, Perú y Venezuela desde diciembre 30 de 1988 hasta junio 5 de 1998.

Otros trabajos se han centrado específicamente en la predicción del comportamiento de los retornos. Alonso y García (2009) buscan predecir la dinámica del IGBC a través de 18 distintos modelos GARCH-M. Las diferentes especificaciones de los modelos usados tuvieron en cuenta el efecto apalancamiento y el efecto día de la semana y hora (que plantea que la distribución de los retornos accionarios difiere en el tiempo).

Sin embargo, no se han realizado estudios que se enfoquen en el comportamiento del mercado bursátil a nivel desagregado a través de modelos de la familia $\mathrm{ARCH}$, y en particular en la existencia del efecto apalancamiento, objetivos del presente trabajo.

\section{Resultados}

Se usó la base de datos de la Bolsa de Valores de Colombia para obtener las series que son objeto de análisis. La primera serie tenida en cuenta es el Índice General de la Bolsa de Valores que recoge el comportamiento de las acciones más representativas de Colombia en función de su rotación y frecuencia. Se tendrán en cuenta un total de 2.194 observaciones diarias: desde el 2 de enero de 2006 hasta el 30 de diciembre de 2014. Adicionalmente, se tendrá en cuenta en el análisis el COLCAP, que es un indicador que refleja la dinámica de las 20 acciones más líquidas de la Bolsa de Valores de Colombia, este índice reemplazó al IGBC como el principal indicador del comportamiento del mercado accionario colombiano. Se tienen 1.704 observaciones diarias recogidas durante el período comprendido entre el 15 de enero del 2008 y el 30 de diciembre de 2014. A continuación, se presenta el comportamiento de las dos series y sus respectivas variaciones porcentuales:

\section{Figura 1.}

\section{IGBC y su variación}


Fuente: Bolsa de Valores de Colombia 
- Figura 2.

\section{Índice COLCAP y su variación}

COLCAP
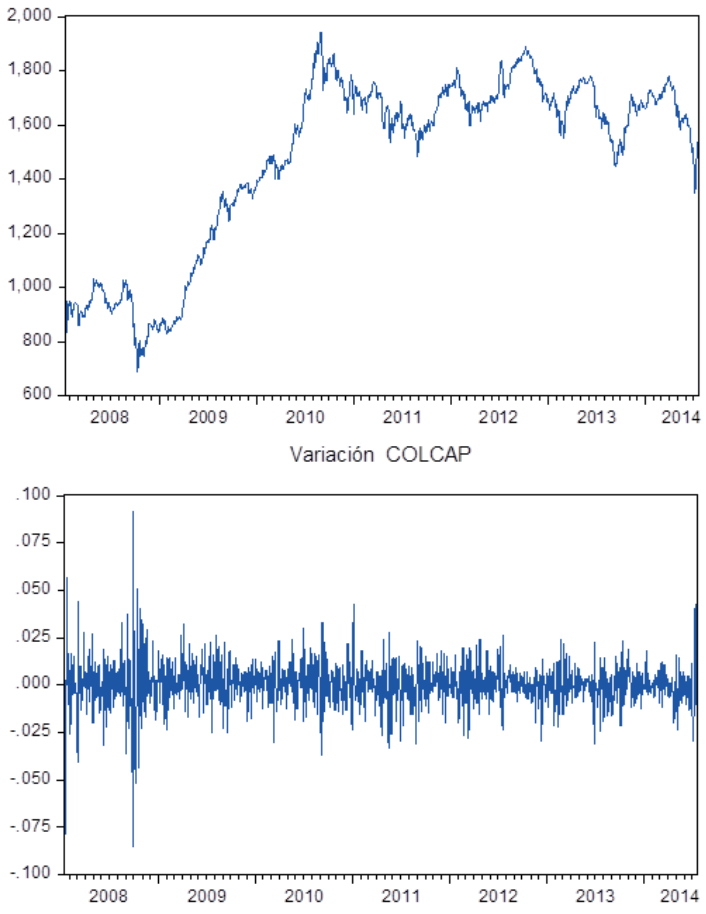

Fuente: Bolsa de Valores de Colombia

Se observa que el IGBC y el índice COLCAP tienen una tendencia bastante similar (creciente hasta el año 2010, desde cuando parece haber cierta estabilidad).

Para realizar un análisis a nivel desagregado se eligieron seis acciones para analizar sus rendimientos $^{1}$ : Bancolombia, Cementos ARGOS, Empresa de Telecomunicaciones de Bogotá, Grupo AVAL, Banco de Bogotá y Almacenes ÉXITO. Para la elección se tuvieron en cuenta aquellas acciones que han estado presentes en el cálculo del IGBC siendo las más representativas del mercado accionario colombiano, la disponibilidad de datos desde el 2006 hasta el
2014 fue de igual forma determinante a la hora de la elección. A continuación se presentan los gráficos de los retornos de las seis acciones:

\section{Figura 3.}

\section{Retornos de las seis acciones elegidas}
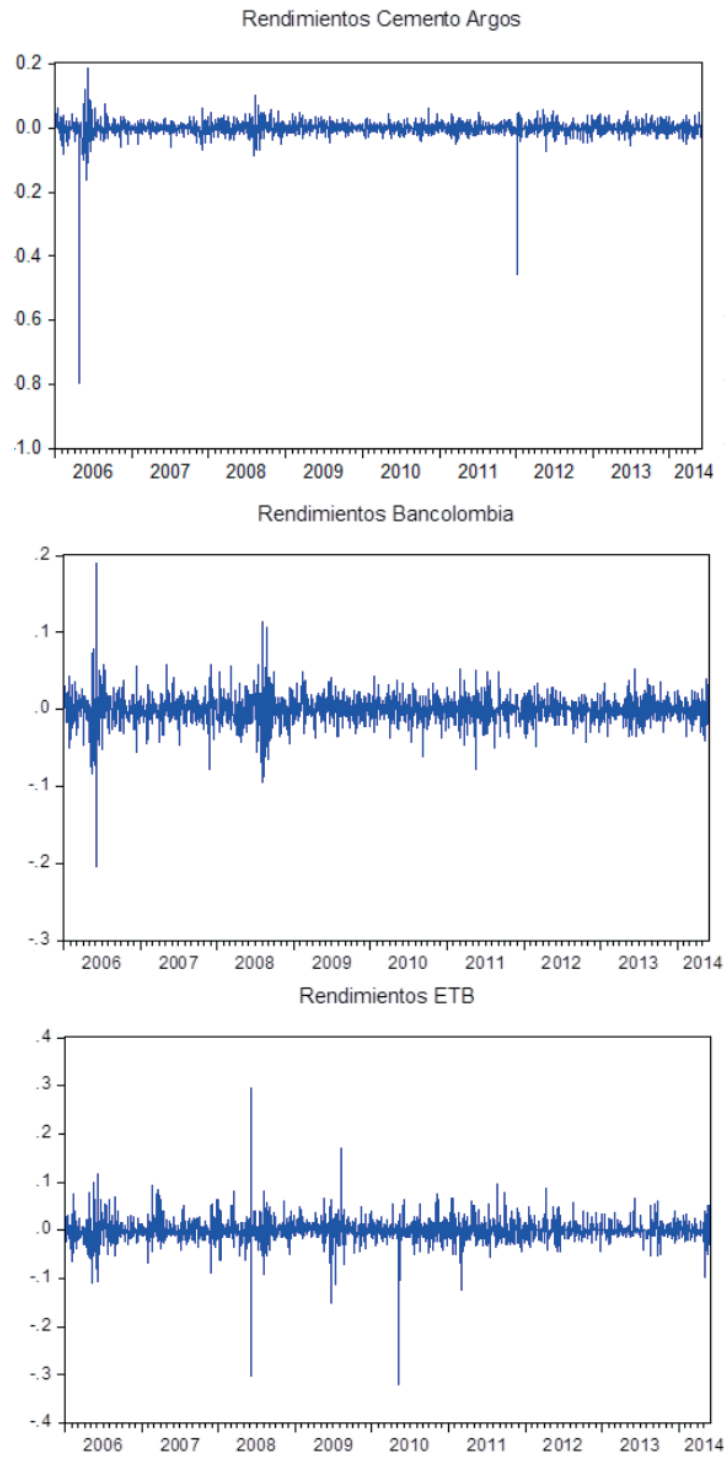

1. Se definen los rendimientos como el cambio en los logaritmos de los precios de las acciones: $r_{i t}=\ln P_{i t}-\ln P_{i t-1}$. Para la serie de rendimientos de cementos Argos, ya que presenta valores atípicos se usó la siguiente transformación: $r_{i t}=\frac{P_{t}-P t-1}{P t-1}$ 
$\longrightarrow$ Continuación de la Figura 3.
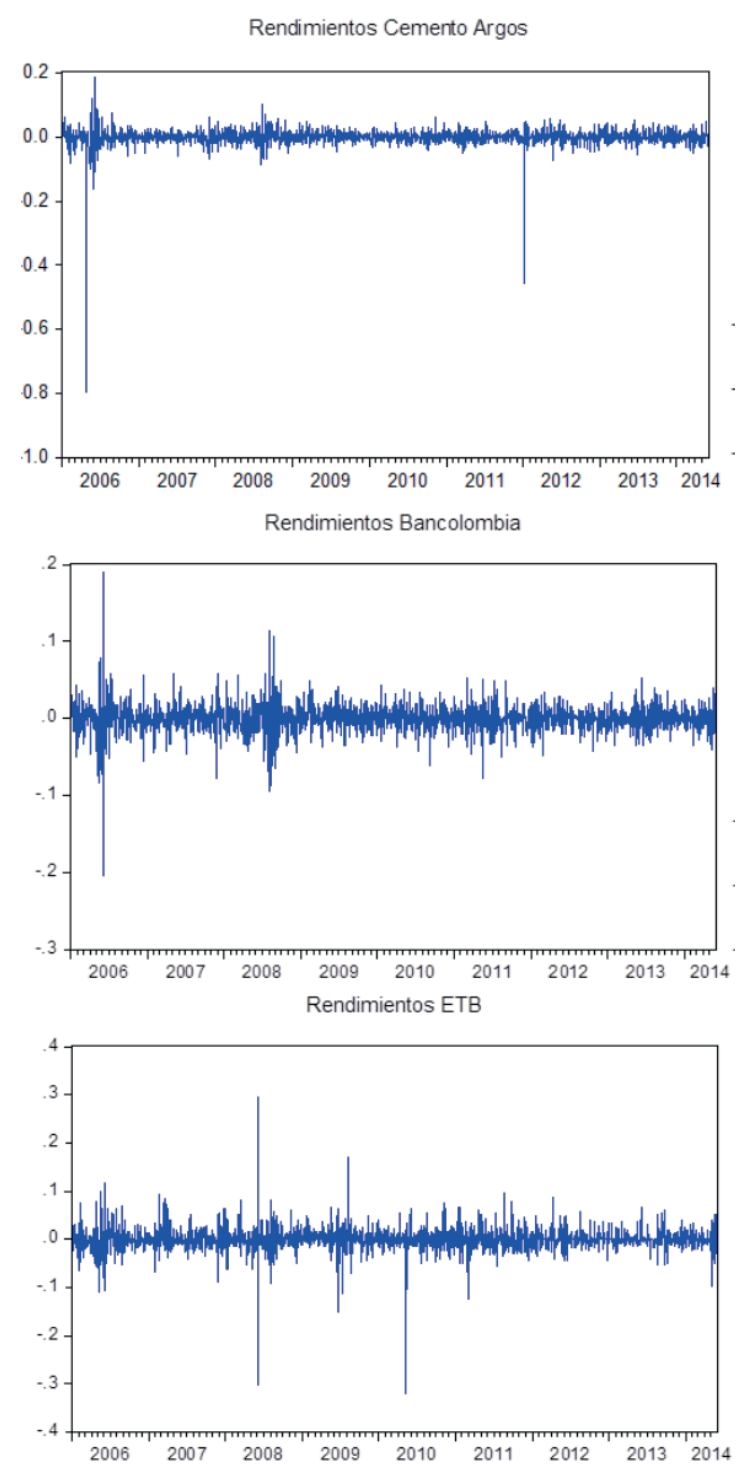

Fuente: Bolsa de Valores de Colombia

Para la serie de Argos hay dos períodos con rendimientos que indican valores muy bajos: específicamente el 28 de abril de 2006 y el 18 de agosto de 2011. En el caso del valor registrado para abril de 2006, el nivel tan bajo se debe a que el día 28 de dicho mes entró en vigencia la disminución del valor de la acción con aumento del número de acciones en circulación, esto como resultado de un proceso de integración de las siete cementeras que conforman el grupo Argos. El dato atípico del 18 de agosto de 2011 por su parte, está relacionado con la compra por parte de Argos de varios activos de la firma Lafarge.

Se procede ahora a presentar algunas estadísticas. La tabla 1 presenta las estadísticas descriptivas de los rendimientos de los activos para el período comprendido entre el 2 de enero de 2006 y el 30 de marzo de 2014, anexo a las pruebas descriptivas se realiza el test de normalidad Jarque-Bera y se muestra tanto el estadístico como el $p$-value correspondiente.

Se puede observar que todas las series de rendimientos presentan un marcado componente asimétrico y una curtosis mucho mayor a tres, lo que sugiere leptocurtosis, indicando una distribución apuntada respecto a la distribución normal. Las series COLCAP, Éxito, Banco de Bogotá, Bancolombia, Cementos Argos y ETB presentan un sesgo negativo mientras que IGBC y AVAL muestran un coeficiente de asimetría positivo. Estos resultados expresan que las series de rendimientos que están siendo analizadas no son simétricas ni tienen curtosis bajas (menores a tres), por tanto no se ajustan a una distribución normal como se confirma a partir del test de Jarque-Bera. El análisis anterior justifica la conveniencia de implementar modelos de la familia GARCH, en especial aquellos que logren describir con mayor acierto el componente asimétrico de las series.

Se inicia realizando un test de efectos ARCH que pone a prueba la hipótesis nula de que no hay efectos ARCH. La ecuación especificada para la media de cada retorno se eligió de acuerdo al criterio AIC y fue en su mayoría un modelo AR(1) sin intercepto. Para todos los casos, la hipótesis nula es rechazada, por lo tanto, es conveniente usar modelos de varianza condicional para modelar el comportamiento del mercado de acciones.

Inicialmente, se decidió estimar los modelos $A R(1)$ $\operatorname{TGARCH}(1,1)$ y $A R(1)-\operatorname{EGARCH}(1,1)$ para cada una de las ocho series que se están analizando mediante el procedimiento de estimación presentado por Porras y Valdés (2011), para obtener un total de 16 modelos. En la mayoría de los mismos, como se aprecia en las tablas 2 y 3,16 de los 18 modelos muestran que el intercepto de la ecuación de la media $\phi_{0}$ resulta 
no ser significativo con un $95 \%$ de confianza. Se evidencia que los modelos TGARCH y EGARCH son similares en su estructura. Adicionalmente se estimó la misma ecuación para la media (un modelo
$A R(1))$ y por tanto solo se hizo necesario estimar los parámetros $\phi_{0}$ y $\phi_{1}$. Al evaluar los signos y la significancia de los parámetros es posible examinar la conveniencia y validez de cada modelo.

-Tabla 1.

\section{Estadísticas descriptivas de los rendimientos de las acciones más representativas en Colombia}

\begin{tabular}{|l|c|c|c|c|c|c|}
\hline & Media & Desv. Estándar & Coef. Asimetría & Curtosis & Jarque-Bera & P-Value \\
\hline IGBC & 0,0001 & 0,0138 & 0,0553 & 15,2830 & 21238,94 & $-0,2207$ \\
\hline COLCAP & 0,0003 & 0,0113 & $-0,2207$ & 11,2605 & 4847,1730 & 0,0000 \\
\hline Bancolombia & 0,0002 & 0,0182 & $-0,1586$ & 15,8379 & 22817,4700 & 0,0000 \\
\hline Cemento Argos & $-0,0006$ & 0,0416 & $-27,4957$ & 1022,7297 & 95416446,0000 & 0,0000 \\
\hline ETB & $-0,0003$ & 0,0245 & $-0,9123$ & 36,7604 & 123200,9000 & 0,0000 \\
\hline AVAL & 0,0003 & 0,0117 & 0,1686 & 11,2916 & 6297,5890 & 0,0000 \\
\hline Éxito & 0,0006 & 0,0181 & $-0,1260$ & 11,2961 & 6297,5890 & 0,0000 \\
\hline Bogotá & 0,0004 & 0,0147 & $-0,2043$ & 14,9870 & 13151,1100 & 0,0000 \\
\hline
\end{tabular}

Fuente: Elaboración propia.

La tabla 2 muestra la estimación del modelo AR(1)$\operatorname{EGARCH}(1,1)$. Se observa que para seis modelos el intercepto de la ecuación de la media no es significativo con un $95 \%$ de confianza, lo mismo sucede con el coeficiente del parámetro autorregresivo, que resulta ser no significativo para cinco de los modelos. Respecto a la ecuación de la varianza todos los parámetros son significativos para las ocho series.

Teniendo en cuenta la construcción teórica del modelo, como ya se indicó anteriormente, si $\gamma_{i}<0$ existe efecto apalancamiento en la serie, si $\gamma_{i}=0$ se puede establecer que no hay asimetría en el modelo. A partir de estos criterios se observa que para las ocho series el coeficiente $\gamma_{\dot{j}}$ resulta significativo y siete de ellas presentan efecto apalancamiento, solo para la serie de rendimientos de las acciones de cementos Argos no hay tal efecto, ya que $\gamma>0$, así las malas noticias reducirían la volatilidad. Hay que tener en cuenta que, para la ecuación de la media de rendimientos de Cementos Argos no se tuvieron en cuenta los dos datos extremos en la serie de rendimientos, sobre esto se discutirá más adelante.
Respecto al modelo AR(1)-TGARCH(1,1) si $\gamma_{i}>0$ las malas noticias incrementan la volatilidad dando lugar al efecto apalancamiento. Para la ecuación de la media, el intercepto resulta no ser significativo en ninguno de los ocho modelos y $\phi_{1}$ es significativamente distinto de cero en tres de ellas. Para la ecuación de la varianza todos los parámetros son significativos y se observa efecto apalancamiento en las ocho series, ya que en todas $\gamma_{i}>0$.

Se revisaron los siguientes supuestos para los residuos: no autocorrelación, normalidad, heterocedasticidad y estacionariedad. Ninguno de los modelos AR(1)-EGARCH(1,1) y AR(1)-TGARCH(1,1) cumple con los supuestos de normalidad y heterocedasticidad, para este último modelo, aplicado a la serie Argos tampoco se cumple incorrelación.

Con el fin de hacer más eficientes los modelos se presentan nuevamente 8 estimaciones de los modelos EGARCH y 8 estimaciones de los modelos TGARCH con algunas variaciones respecto a lo realizado por Porras y Valdés (2011), para tal efecto, en la ecuación de la media se suprimió $\phi_{0}$ en los casos donde 
resulta ser no significativo y se mantuvo un $A R(1)$ al ser en todos los casos el mejor modelo según el criterio AIC. Para la varianza se mantuvo el modelo
$\operatorname{GARCH}(1,1)$ por ser más parsimonioso, evitando un sobre ajuste haciendo que sea menos probable obtener estimaciones negativas.

Tabla 2.

Parámetros estimados y $p$-value del modelo $\operatorname{AR}(1)-\operatorname{EGARCH}(1,1)$ asociados a estadísticos $t$

\begin{tabular}{|l|c|c|c|c|c|c|c|c|c|c|c|c|}
\hline & \multicolumn{2}{|c|}{$\phi_{0}$} & \multicolumn{2}{|c|}{$\phi_{1}$} & \multicolumn{2}{|c|}{$\alpha_{0}$} & \multicolumn{2}{|c|}{$\alpha_{1}$} & \multicolumn{2}{|c|}{$\gamma$} & \multicolumn{2}{|c|}{$\beta$} \\
\hline $\begin{array}{l}\text { Acciones } \\
\text { colombianas }\end{array}$ & coef & prob & coef & prob & coef & prob & coef & prob & coef & prob & coef & prob \\
\hline Argos & $-0,01$ & 0,00 & $-0,09$ & 0,00 & $-6,70$ & 0,00 & 2,30 & 0,00 & 0,50 & 0,00 & 0,24 & 0,00 \\
\hline AVAL & 0,00 & 0,05 & $-0,03$ & 0,12 & $-0,87$ & 0,00 & 0,36 & 0,00 & $-0,06$ & 0,00 & 0,93 & 0,00 \\
\hline Bancolombia & 0,00 & 0,28 & $-0,04$ & 0,09 & $-0,62$ & 0,00 & 0,21 & 0,00 & $-0,08$ & 0,00 & 0,94 & 0,00 \\
\hline Bogotán & 0,00 & 0,35 & $-0,02$ & 0,34 & $-0,82$ & 0,00 & 0,28 & 0,00 & $-0,07$ & 0,00 & 0,93 & 0,00 \\
\hline EIB & 0,00 & 0,86 & $-0,01$ & 0,76 & $-1,01$ & 0,00 & 0,22 & 0,00 & $-0,02$ & 0,00 & 0,88 & 0,00 \\
\hline Éxito & 0,00 & 0,10 & 0,04 & 0,08 & $-2,31$ & 0,00 & 0,35 & 0,00 & $-0,07$ & 0,00 & 0,75 & 0,00 \\
\hline COLCAP & 0,00 & 0,24 & 0,08 & 0,00 & $-0,84$ & 0,00 & 0,28 & 0,00 & $-0,09$ & 0,00 & 0,93 & 0,00 \\
\hline IGBC & 0,00 & 0,63 & 0,12 & 0,00 & $-0,92$ & 0,00 & 0,33 & 0,00 & $-0,12$ & 0,00 & 0,93 & 0,00 \\
\hline
\end{tabular}

Fuente: Elaboración propia.

Tabla 3.

Parámetros estimados y $\mathrm{p}$-value del modelo $\operatorname{AR}(1)-\operatorname{TGARCH}(1,1)$ asociados a estadísticos $\mathrm{t}$

\begin{tabular}{|c|c|c|c|c|c|c|c|c|c|c|c|c|}
\hline \multirow[b]{2}{*}{$\begin{array}{l}\text { Acciones } \\
\text { colombianas }\end{array}$} & \multicolumn{2}{|c|}{$\phi_{0}$} & \multicolumn{2}{|c|}{$\phi_{1}$} & \multicolumn{2}{|c|}{$\alpha_{0}$} & \multicolumn{2}{|c|}{$\alpha_{1}$} & \multicolumn{2}{|c|}{$r$} & \\
\hline & coef & prob & coef & prob & coef & prob & coef & prob & coef & prob & coef & prob \\
\hline Argos & $-0,0003$ & 0,8816 & $-0,0021$ & 0,9504 & 0,0007 & 0,0000 & $-0,0248$ & 0,0000 & 0,0241 & 0,0000 & 0,5829 & 0,0000 \\
\hline AVAL & 0,0004 & 0,1566 & $-0,0356$ & 0,1218 & $2,01 \mathrm{E}-05$ & 0,0000 & 0,1647 & 0,0000 & 0,1458 & 0,0000 & 0,7163 & 0,0000 \\
\hline Bancolombia & 0,0003 & 0,3794 & $-0,0428$ & 0,0668 & $3,73 \mathrm{E}-05$ & 0,0000 & 0,1097 & 0,0000 & 0,1363 & 0,0000 & 0,6871 & 0,0000 \\
\hline Bogotá & $3,80 \mathrm{E}-06$ & 0,9869 & $-0,0310$ & 0,1978 & $1,33 \mathrm{E}-05$ & 0,0000 & 0,1117 & 0,0000 & 0,1059 & 0,0000 & 0,7666 & 0,0000 \\
\hline ETB & $-4,55 E-05$ & 0,9318 & 0,0252 & 0,3433 & 0,0002 & 0,0000 & 0,0882 & 0,0000 & 0,1789 & 0,0000 & 0,5769 & 0,0000 \\
\hline Éxito & 0,0003 & 0,4173 & 0,0503 & 0,0372 & 0,0001 & 0,0000 & 0,1377 & 0,0000 & 0,1336 & 0,0000 & 0,6392 & 0,0000 \\
\hline
\end{tabular}

Fuente: Elaboración propia.

Los resultados para el modelo $\mathrm{AR}(1)-\operatorname{EGARCH}(1,1)$ se observan en la Tabla 4. Para los rendimientos de las series AVAL y Éxito se incluyó intercepto en la ecuación de la media, ya que resulta ser significativo con un $90 \%$ de confianza. Para la serie de rendimientos de cementos Argos se incluyeron dos variables pulso en las fechas donde se presentan observaciones extremas con el fin de modelar el efecto de estos datos atípicos presentes en la serie. Los resultados indican que, para tres de los 
modelos, $\phi_{1}$ no es significativo con un nivel de confianza del $90 \%$. En la ecuación de la varianza todos los términos siguen siendo significativos con un $90 \%$ de confianza y en todos ellos $\gamma_{i}<0$, lo que señala que en todas las series se presenta efecto apalancamiento. Como caso particular, dado que en la media de los rendimientos de Cementos
Argos se incluyeron las dos variables dummies para los datos atípicos, el parámetro $\gamma_{i}$ pasó de ser positivo a ser negativo, indicando la presencia de efecto apalancamiento. Se resalta además que si se asume un nivel de confianza del 95\%, la serie ETB no presentaría apalancamiento pues el parámetro $\gamma$ no sería significativo.

\section{TTabla 4.}

\section{Parámetros estimados y $p$-value del modelo AR(1)-EGARCH(1,1) asociados a estadísticos $t$}

\begin{tabular}{|c|c|c|c|c|c|c|c|c|c|c|c|c|c|c|c|c|}
\hline & \multicolumn{2}{|c|}{ Dummy 1} & \multicolumn{2}{|c|}{ Dummy 2} & \multicolumn{2}{|c|}{$\phi_{0}$} & \multicolumn{2}{|c|}{$\phi_{1}$} & \multicolumn{2}{|c|}{$\alpha_{0}$} & \multicolumn{2}{|c|}{$\alpha_{1}$} & \multicolumn{2}{|c|}{$\gamma$} & \multicolumn{2}{|c|}{$\beta$} \\
\hline $\begin{array}{l}\text { Acciones } \\
\text { colombianas }\end{array}$ & coef & prob & coef & prob & coef & prob & coef & prob & coef & prob & coef & prob & coef & prob & coef & prob \\
\hline Argos & $-1,60$ & 0,00 & $-0,63$ & 0,00 & 0,00 & 0,08 & 0,01 & 0,75 & $-1,11$ & 0,00 & 0,33 & 0,00 & $-0,07$ & 0,00 & 0,89 & 0,00 \\
\hline AVAL & - & - & - & - & 0,00 & 0,05 & $-0,03$ & 0,12 & $-0,87$ & 0,00 & 0,36 & 0,00 & $-0,06$ & 0,00 & 0,93 & 0,00 \\
\hline Bancolombia & - & - & - & - & - & - & $-0,33$ & 0,10 & $-0,62$ & 0,00 & 0,21 & 0,00 & $-0,08$ & 0,00 & 0,94 & 0,00 \\
\hline Bogotá & - & - & - & - & - & - & $-0,02$ & 0,25 & $-0,80$ & 0,00 & 0,28 & 0,00 & $-0,07$ & 0,00 & 0,93 & 0,00 \\
\hline ETB & - & - & - & - & - & - & 0,00 & 0,96 & $-1,02$ & 0,00 & 0,22 & 0,00 & $-0,02$ & 0,09 & 0,88 & 0,00 \\
\hline Éxito & - & - & - & - & 0,00 & 0,10 & 0,04 & 0,08 & $-2,31$ & 0,00 & 0,35 & 0,00 & $-0,07$ & 0,00 & 0,75 & 0,00 \\
\hline COLCAP & - & - & - & - & - & - & 0,09 & 0,00 & $-0,84$ & 0,00 & 0,28 & 0,00 & $-0,10$ & 0,00 & 0,93 & 0,00 \\
\hline IGBC & - & - & - & - & - & - & 0,12 & 0,00 & $-0,92$ & 0,00 & 0,33 & 0,00 & $-0,12$ & 0,00 & 0,93 & 0,00 \\
\hline
\end{tabular}

Fuente: Elaboración propia.

La tabla 5 muestra los resultados de la estimación del modelo AR(1)-TGARCH(1,1). Como se observa para ninguna serie se estimó $\phi_{0}$ y el parámetro $\phi_{1}$ resulta no ser significativo para cuatro de las series con un $90 \%$ de confianza. Adicionalmente, en la ecuación de la varianza todos los parámetros resultan ser significativamente distintos de cero, incluyendo los relativos al efecto apalancamiento. Por otro lado, el parámetro $\gamma_{i}$ es positivo, razón por la cual es posible concluir a partir de este modelo que las seis acciones estudiadas sumadas al índice COLCAP e IGBC presentan efecto apalancamiento.

Para este segundo par de modelos se examina igualmente el cumplimiento de supuestos. Sin embargo, no se observan mayores variaciones respecto a la primera especificación (el modelo TGARCH de rendimientos Argos cumple en este caso el supuesto de no autocorrelación)
La tabla 6 permite hacer una comparación de los estimadores de bondad de ajuste a partir de los criterios Akaike y log-likelihood para los segundos modelos TGARCH y EGARCH. Para establecer el mejor modelo se comparan los estimadores akaike y log-likelihood, para el caso AIC es mejor el modelo que presente un menor valor, contrario al criterio de log-likelihood donde es mejor el modelo con mayor valor de estimación. Se puede observar que tanto el modelo TGARCH como el EGARCH presentan un buen ajuste para las series de las acciones colombianas que están siendo analizadas, tanto si se toman a nivel desagregado (una acción) 0 a nivel agregado (tomando la totalidad del mercado), esto a raíz de que los valores para los dos criterios son muy similares. Se puede, sin embargo, destacar que bajo el criterio Akaike resulta tener mejor ajuste el modelo EGARCH para las series Bancolombia, Bogotá y Éxito; mientras que las series ARGOS y COLCAP 
presentan mejor ajuste con el modelo TGARCH. Si se tiene en cuenta el criterio log-likelihood las series Bancolombia, Bogotá, Éxito, IGBC y ARGOS son mejor ajustadas por un modelo TGARCH, mientras que AVAL, ETB y COLCAP se ajustan adecuadamente con un modelo EGARCH.

Tabla 5.

Parámetros estimados y $p$-value del modelo $\operatorname{AR}(1)-\operatorname{TGARCH}(1,1)$ asociados a estadísticos $t$

\begin{tabular}{|l|c|c|c|c|c|c|c|c|c|c|c|c|c|c|}
\hline & \multicolumn{2}{|c|}{ Dummy 1 } & \multicolumn{2}{|c|}{ Dummy 2 } & \multicolumn{2}{|c|}{$\phi_{0}$} & \multicolumn{2}{|c|}{$\alpha_{0}$} & \multicolumn{2}{|c|}{$\alpha_{1}$} & \multicolumn{3}{|c|}{$\gamma$} & \multicolumn{2}{c|}{$\beta$} \\
\hline $\begin{array}{l}\text { Acciones } \\
\text { colombianas }\end{array}$ & coef & prob & coef & prob & coef & prob & coef & prob & coef & prob & coef & prob & coef & prob \\
\hline Argos & $-0,59$ & 0,00 & $-1,60$ & 0,86 & 0,00 & 0,89 & 0,00 & 0,00 & 0,12 & 0,00 & 0,15 & 0,00 & 0,69 & 0,00 \\
\hline AVAL & - & - & - & - & $-0,03$ & 0,14 & 0,00 & 0,00 & 0,16 & 0,00 & 0,16 & 0,00 & 0,71 & 0,00 \\
\hline Bancolombia & - & - & - & - & $-0,04$ & 0,07 & 0,00 & 0,00 & 0,11 & 0,00 & 0,14 & 0,00 & 0,69 & 0,00 \\
\hline Bogotá & - & - & - & - & $-0,03$ & 0,19 & 0,00 & 0,00 & 0,11 & 0,00 & 0,11 & 0,00 & 0,77 & 0,00 \\
\hline ETB & - & - & - & - & 0,03 & 0,34 & 0,00 & 0,00 & 0,09 & 0,00 & 0,18 & 0,00 & 0,58 & 0,00 \\
\hline Éxito & - & - & - & - & 0,05 & 0,03 & 0,00 & 0,00 & 0,14 & 0,00 & 0,14 & 0,00 & 0,64 & 0,00 \\
\hline COLCAP & - & - & - & - & 0,10 & 0,00 & 0,00 & 0,00 & 0,07 & 0,00 & 0,16 & 0,00 & 0,77 & 0,00 \\
\hline IGBC & - & - & - & - & 0,12 & 0,00 & 0,00 & 0,00 & 0,08 & 0,00 & 0,22 & 0,00 & 0,74 & 0,00 \\
\hline
\end{tabular}

Fuente: Elaboración propia.

Tabla 6.

Estimadores de Bondad y ajuste de modelos AR(1)-TGARCH y AR(1)-EGARCH

\begin{tabular}{|l|c|c|c|c|} 
& \multicolumn{2}{|c|}{$\operatorname{AR}(1)-\operatorname{TGARCH}(1,1)$} & \multicolumn{2}{c|}{ AR(1)-EGARCH(1,1) } \\
\hline Acciones colombianas & logl & Akaike & logl & Akaike \\
\hline Argos & 5.882 .725 & -5.358 .619 & 5.867 .601 & $-5,343914$ \\
\hline AVAL & 6.143 .041 & -5.597 .848 & 6.144 .211 & $-5,598004$ \\
\hline Bancolombia & 6.025 .175 & -5.490 .356 & 6.016 .994 & $-5,482895$ \\
\hline Bogotá & 6.597 .986 & -6.012 .755 & 6.581 .610 & $-5,997821$ \\
\hline ETB & 5.187 .539 & -4.726 .438 & 5.197 .148 & $-4,735201$ \\
\hline Exito & 5.866 .728 & -5.345 .853 & 5.852 .609 & $-5,322065$ \\
\hline COLCAP & 5.506 .964 & -64.766 .709 & 5.512 .528 & $-6,483259$ \\
\hline IGBC & 6.837 .065 & -6.233 .636 & 6.834 .656 & $-6,231438$ \\
\hline
\end{tabular}

Fuente: Elaboración propia. 


\section{Conclusión}

En este trabajo se realiza un ejercicio comparativo a través de la estimación de los modelos no lineales TGARCH y EGARCH para seis de las acciones más representativas del mercado accionario colombiano y el índice COLCAP e IGBC durante el periodo comprendido entre el 2 de enero de 2006 hasta el 30 de diciembre de 2014; esta clase de análisis no se había realizado anteriormente para el comportamiento desagregado de las acciones en Colombia. Al realizar el análisis descriptivo de las series se encuentra que todas presentan altos coeficientes de asimetría, curtosis altas y no se distribuyen normalmente. Adicional a esto, los test de efectos ARCH realizados para las ocho series muestran que es adecuado el uso de modelos de varianza condicional para estudiar el análisis de las series del mercado accionario colombiano.

Después de la estimación de 32 modelos, se encuentra que tanto las series de los retornos como las de los índices presentan efectos apalancamiento, de modo que las malas noticias tienen un impacto mayor en la volatilidad de las series, solamente para el segundo modelo EGARCH estimado la serie de retornos de ETB no se ve afectada por el efecto apalancamiento.

A partir del criterio AIC y la verosimilitud generada por cada modelo se observa que tanto el modelo TGARCH como el EGARCH presentan un buen ajuste para las series de las acciones colombianas que están siendo analizadas, tanto si se toman a nivel desagregado (una acción) 0 a nivel agregado, ya que los valores para los dos criterios son muy similares. Bajo el criterio Akaike resulta tener mejor ajuste el modelo EGARCH para las series Bancolombia, Bogotá y Éxito, mientras que las series ARGOS y COLCAP presentan mejor ajuste con el modelo TGARCH.

Estos resultados señalan la pertinencia de la implementación de modelos de la familia ARCH para evaluar y confirmar finalmente la existencia de efecto apalancamiento en el mercado accionario colombiano, lo que implica que la información tiene efectos asimétricos sobre los mercados bursátiles.
En un futuro sería interesante la implementación de este mismo análisis, pero con un mayor volumen de acciones.

Finalmente, es de resaltar la existencia reciente de metodologías o líneas de investigación que se enfocan en el análisis de series de tiempo con características de volatilidad que involucran modelos que dan respuestas dinámicas a variables de estudio que son alteradas por choques en el sistema 0 choques en variables exógenas mediante el análisis de funciones de impulso respuesta. Estos enfoques corresponden a modelos GARCH multivariados, en Colombia hay muy poca investigación que involucre estos modelos, por lo que sería de interés orientar investigaciones profundas en el análisis de series de tiempo financieras, implementando metodologías que enriquezcan las herramientas de descripción y pronósticos de este tipo de series. A pesar de que los modelos GARCH multivariados amplían con eficiencia las estimaciones sobre series que presentan gran volatilidad, mantienen una baja implementación entre otras cosas porque son pocos los software especializados para tal fin, dado que como se ha visto, el costo computacional para este tipo de modelos resulta ser alto la mayoría de las veces, sin embargo, se han logrado algunos avances (Ehlers \& Fioruci, 2014).

\section{Referencias Bibliográficas}

Alonso, J., \& García, J. (2009). ¿Qué tan buenos son los patrones del IGBC para predecir su comportamiento? una aplicación con datos de alta frecuencia. Universidad ICECI, Estudios Generales, vol 25, No. 112. Recuperado de: http:// www.icesi.edu.co/departamentos/economia/publicaciones/docs/BORRAEC0/borrador_14_prediccion_igbc.pdf

Arjona, E \& Ortiz, E. (2001). Heterokedastic Behavior of the Latin American Emerging Stock Markets. International Review of Financial Analysis, v. 10, iss. 3, pp. 287-305

Bollerslev, T. (1986). Generalized autoregressive conditional heteroscedasticity. Journal of Econometrics, 31(3), 307-327

Casas, M., \& Cepeda, E. (2008). Modelos ARCH, GARCH y EGARCH: Aplicaciones a series financieras, Cuadernos de economía, Bogotá. Recuperado de: http://www.sfcielo.org. co/pdf/ceco/v27n48/v27n48a11.pdf 
Efecto apalancamiento en el mercado accionario colombiano

Charris, A. García, R., \& Parody E. (2012). Modelación de la volatilidad y pronóstico del índice general de la bolsa de valores de Colombia (IGBC). Revista Clío América Universidad de Magdalena. Vol. 6, núm. 12. Recuperado de http:// revistas.unimagdalena.edu.co/index.php/clioamerica/ article/view/432

Ehlers, R., \& Fioruci, J. (2014). BayesDccGarch- An implementation of Multivariate GARCH DCC Models, Cornell University library, Brasil. Recuperado de http://arxiv.org/ pdf/1412.2967.pdf

Fernández, H., \& Pérez, F. (2006). Análisis de la volatilidad del İ́ndice General de la Bolsa de Valores de Colombia utilizando un modelo ARCH. Revista de Ingenierías universidad de Medellín. vol. 5, núm. 8, Recuperado de: http://www. redalyc.org/pdf/750/75050803.pdf

Grajales, C., \& Pérez, 0. (2008). Modelos discretos y continuos para estimar la densidad de probabilidad de la volatilidad estocástica de los rendimientos de series financieras. Cuadernos de Administración. Pontificia Universidad Javeriana, vol. 21, núm. 36 Colombia. Recuperado de http://www.redalyc.org/pdf/205/20503606.pdf

Haque, M., Hassan, M., \& Varela, 0. (2001). Stability, Volatility, Risk Premiums, and Predictability in Latin American Emerging Stock Markets. Quarterly Journal of Business and Economics Vol. 40, No. 3/4 (Summer - Autumn, 2001), pp. 23-44. Recuperado de http://www.jstor.org/ stable/40473332

Nelson, D. (1991). Conditional heterosedasticity in asset returns: A new approach, Econometrica, 347-370. Recuperado de http://www.samsi.info/sites/default/files/ Nelson_1991.pdf

Porras, A., \& Valdés A. (2011). Modelación de los rendimientos bursátiles mexicanos mediante los modelos TGARCH y EGARCH: un estudio econométrico para 30 acciones y el Índice de Precios y Cotizaciones. Universidad de Guadalajara. MPRA Paper No. 36872. Recuperado de: http://mpra.ub.uni-muenchen.de/36872/1/MPRA_paper_36872.pdf

Porras, A., \& Valdés, (2014) A TGARCH model with an asimmetric Student's t distribution an the rationality hypotheses of stock investors in Latin America. MPRA. Recuperado de: http://mpra.ub.uni-muenchen.de/53019/1/MPRA_paper_53019.pdf

Reyes, 0., \& Sánchez, A. (2006). Regularidades probabilísticas de las series financieras y la familia de los modelos GARCH, Universidad Autónoma del Estado de México. Recuperado de http://www.redalyc.org/articulo.oa?id=10413205 